\title{
Impact of Cholesterol on Voids in Phospholipid Membranes
}

\author{
Emma Falck \\ Laboratory of Physics and Helsinki Institute of Physics, \\ Helsinki University of Technology, P. O. Box 1100, FI-02015 HUT, Finland \\ Michael Patra and Mikko Karttunen \\ Biophysics and Statistical Mechanics Group, Laboratory of Computational Engineering, \\ Helsinki University of Technology, P. O. Box 9203, FI-02015 HUT, Finland \\ Marja T. Hyvönen \\ Wihuri Research Institute, Kalliolinnantie 4, FI-00140 Helsinki, \\ Finland, and Laboratory of Physics and Helsinki Institute of Physics, \\ Helsinki University of Technology, P. O. Box 1100, FI-02015 HUT, Finland \\ Ilpo Vattulainen \\ Laboratory of Physics and Helsinki Institute of Physics, \\ Helsinki University of Technology, P. O. Box 1100, FI-02015 HUT, Finland
}

(Dated: November 6, 2018)

\begin{abstract}
Free volume pockets or voids are important to many biological processes in cell membranes. Free volume fluctuations are a prerequisite for diffusion of lipids and other macromolecules in lipid bilayers. Permeation of small solutes across a membrane, as well as diffusion of solutes in the membrane interior are further examples of phenomena where voids and their properties play a central role. Cholesterol has been suggested to change the structure and function of membranes by altering their free volume properties. We study the effect of cholesterol on the properties of voids in dipalmitoylphosphatidylcholine (DPPC) bilayers by means of atomistic molecular dynamics simulations. We find that an increasing cholesterol concentration reduces the total amount of free volume in a bilayer. The effect of cholesterol on individual voids is most prominent in the region where the steroid ring structures of cholesterol molecules are located. Here a growing cholesterol content reduces the number of voids, completely removing voids of the size of a cholesterol molecule. The voids also become more elongated. The broad orientational distribution of voids observed in pure DPPC is, with a $30 \%$ molar concentration of cholesterol, replaced by a distribution where orientation along the bilayer normal is favored. Our results suggest that instead of being uniformly distributed to the whole bilayer, these effects are localized to the close vicinity of cholesterol molecules.
\end{abstract}

\section{INTRODUCTION}

In addition to space occupied by atoms of, e. g., lipid, sterol, and protein molecules, cell membranes ${ }^{1,2,3}$ incorporate free space or free volume. 4.5 .6 .7 .8 .9 .10 Membranes may be thought of as complex porous structures with a varying number of free volume pockets or voids. The voids might assume different sizes, shapes, and orientations. The nature of the voids is dynamic: they can be generated or annihilated by trans-gauche isomerizations in the hydrocarbon tails of lipids molecules, or less frequently, by the movement of whole lipids.

Voids are crucial for dynamic processes in membranes. 10 Local free volume fluctuations in bilayers are supposed to give rise to jump diffusion of the lipids that constitute the membrane bilayer. ${ }^{4,7}$ Another important process where voids play a central role is the diffusion of small solutes within or across membranes. For instance the electron transport in mitochondria and chloroplasts is primarily influenced by the availability of voids for the diffusion of the electron carrying quinone molecule. ${ }^{11}$ Passive transport of water, oxygen, small organic molecules, and small ions to and from the cell across the plasma membrane is an important process involving voids 5.10 .12 .13 .14 .15 .16 Further, general anesthesia might be partially explained by changes in the packing and void dis- tribution of membranes caused by the partitioning and diffusion of anesthetics in membranes. These changes have been suggested to lead to modifications in the lateral pressure profile of the membrane, and will consequently alter the structure and function of the membrane proteins ${ }^{17.18}$ Voids are also important for the interpretation of fluorescence anisotropy measurements. Decreased fluorescence anisotropy caused by the increased mobility of fluorescent probes such as diphenylhexatriene (DPH) or pyrene could be due to generation of voids. ${ }^{10,19}$ Finally, voids are believed to be involved in thinning transitions in smectic membranes. ${ }^{20}$

There have been several attempts to formally describe the effect of voids on the dynamic processes in cell membranes or the simpler one- and multi-component lipid bilayers. The first free volume and free area theories appeared decades ago. 4.7.21 Free area theories in particular have been used for interpreting the mobilities of lipid or sterol molecules in model bilayers 4.7 The basis of these theories is that a particle attempting a jump needs, in addition to a sufficient activation energy, a large enough empty site to jump to. The diffusion coefficient is therefore thought to depend on the ratio of the close-packed area of the diffusing particle and the average free area per particle. ${ }^{4}$

Free area theories have certain shortcomings. They are twodimensional, i. e., it is assumed that the free volume properties 
do not vary significantly in the direction of the bilayer normal. Unfortunately, bilayers are remarkably heterogeneous in the normal direction, and the amount of free volume varies strongly across a bilayer ${ }^{9.22}$ More important, free area theories are mean-field, that is, all predictions are based on the average free area available per lipid. It is impossible to distinguish between very few large voids and numerous tiny ones. Such a distinction should be highly relevant from the point of view of, e.g., solute diffusion; unhindered motion within a substantial void is expected to differ from jumps between isolated voids. Hence, free area theories should not be used for quantitative predictions. Of late, there have been a few attempts to develop more sophisticated free volume theories ${ }^{23.24}$ These analytical approaches have enhanced our understanding of the impact of voids on dynamic processes.

A detailed understanding of the microscopic properties of voids and their role in dynamical processes will, however, not emerge from analytical considerations, but can be acquired using computer simulations. In two early molecular dynamics (MD) studies, Bassolino-Klimas et al $\stackrel{5.25}{5}$ focused on the diffusion mechanisms of benzene molecules in phospholipid bilayers at different temperatures in the fluid phase. The size distribution of voids was used to explain the motion of the benzene molecules. Further, the size distribution was found to depend

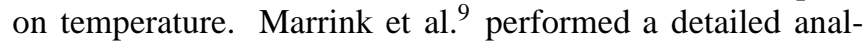
ysis of the properties of voids in a dipalmitoylphosphatidylcholine (DPPC) bilayer in the fluid phase. The size, shape, and orientation of the voids were found to vary significantly in the direction of the bilayer normal. More recently, Shinoda et al ${ }^{26}$ considered the effect of chain branching on the permeability of lipid bilayers, and briefly addressed the issue of voids. They concluded that chain branching would seem to reduce the probability of observing large voids. Jedlovszky et al ${ }^{13}$ have briefly considered the impact of cholesterol on the number of voids in dimyristoylphosphatidylcholine (DMPC) bilayers. In all, computer simulations can be considered a useful tool for studying voids.

In this computational study, we focus on the impact of cholesterol on the properties of voids in lipid bilayers. Cholesterols are rigid molecules that seem to enhance the properties of cell membranes and allow for wider variations in the lipid composition of the membrane.$^{27}$ Important effects of a finite cholesterol concentration on bilayers in the fluid state include changes in passive permeability of small solutes ${ }^{13.24}$ and suppressed lateral diffusion of phospholipids in bilayers containing cholesterol. $1,7,22,28,29,30$ These effects suggest that cholesterol occupies free volume in lipid bilayers ${ }^{4,7,22}$ The void-filling capacity of cholesterol is also thought to be relevant for the formation of membrane domains enriched in glycosphingolipids and cholesterol ${ }^{31}$ Our intention is to study in microscopic detail how the presence of cholesterol molecules influences the properties of voids in phospholipid bilayers.

We have performed $100 \mathrm{~ns}$ MD simulations of DPPC / cholesterol bilayers in the fluid state, with cholesterol concentrations ranging from 0 to $29.7 \mathrm{~mol} \%$ ? 22 The first step in characterizing the voids is to single out the free volume by mapping the bilayer on a rectangular three-dimensional grid and checking which grid points lie outside the van der Waals radii of any atoms in the system. The individual free volume pockets or voids are identified by using a weighted tree-based union/find algorithm with path compression adapted from Ref. 32. Finally, the properties of the voids are analyzed with the aid of principal component analysis, ${ }^{33}$ a technique that yields information on the shape and orientation of voids. Combining these methods, we investigate the effects of cholesterol, permeant size, and location in the bilayer on the properties of voids. We first focus on the size distribution of voids, considering also percolation of free volume. The shape and orientation of voids, and the possible size dependence of these properties, are also examined. Finally, we look into local effects on voids in the close vicinity of cholesterol molecules.

These studies are qualitative rather than quantitative in nature. Our intention is to identify trends and describe new phenomena, simultaneously attempting to shed light on the microscopic reasons behind them.

Our results suggest that replacing phospholipid molecules by cholesterols reduces the total volume of the bilayer. In addition, both the total free volume and the free volume fraction decrease with an increased cholesterol content. As for the void distribution, the effects of cholesterol on the properties of voids are most prominent in the region where the rigid steroid ring parts of cholesterol molecules reside. Other parts of the bilayer are affected to a lesser degree. In the region with the ring structures, the presence of cholesterol reduces the number of voids, completely removing voids of its own size. The voids become more elongated and instead of a broad orientational distribution, which is observed in pure phospholipid bilayers, with $29.7 \%$ cholesterol voids are preferentially oriented along the bilayer normal. We also find that the effect of cholesterol is local, i. e., the effects are most apparent in the close vicinity of cholesterol molecules.

\section{MODEL AND SIMULATION DETAILS}

The model systems we studied are fully hydrated lipid bilayers consisting of 128 macromolecules, i.e., DPPCs and cholesterols, and 3655 water molecules. In this contribution we focus on a pure DPPC bilayer and DPPC/cholesterol bilayers with five different cholesterol molar fractions: $\chi=$ $0 \%, 4.7 \%, 12.5 \%, 20.3 \%$, and $29.7 \%$. The underlying MD simulations have been described and validated elsewhere, ${ }^{22}$ and in the following we shall merely summarize the most important details. The previously published results have been shown to be in good agreement with experimental studies ${ }^{22}$

The united atom force fields for the DPPC and cholesterol molecules were adopted from earlier studies ${ }^{34,35,36}$ As an initial configuration for the pure DPPC bilayer we used the final structure of run E in Ref. 36. For systems with finite cholesterol concentrations, the initial configurations were constructed by replacing randomly selected DPPC molecules with cholesterols such that same number of DPPC molecules was replaced in each monolayer. To fill the free volume left by replacing DPPC molecules by the somewhat smaller cholesterols, the system was thoroughly equilibrated, see Ref. 22 
for details. The bilayer was aligned such that it lies in the xy plane. The bilayer normal is thus parallel to the $\mathrm{z}$-axis.

The MD simulations were run at a temperature $T=323 \mathrm{~K}$ using the GROMACS ${ }^{37}$ molecular simulation software. After the initial equilibration, the system was kept in the $N p T$ ensemble at a pressure of 1 bar using the Berendsen barostat and thermostat 38 The duration of each simulation was $100 \mathrm{~ns}$ and the time step was chosen to be $2.0 \mathrm{fs}$. For long-range electrostatic interactions we used the Particle-Mesh Ewald (PME) method ${ }^{39}$ which has been shown to yield reliable results for bilayers ${ }^{40.41}$

\section{METHODS}

The details of the technique outlined in this section, along with a computer code, will be published elsewhere ${ }^{42}$

\section{A. Finding Free Volume: Grid Approach}

To locate the free volume, the bilayers were mapped onto three-dimensional rectangular grids, an approach outlined in Ref. 9. The grid elements outside the van der Waals radii of any atomic groups were designated as free volume and those inside as occupied volume. The van der Waals radius of a given atomic group was taken to be half of the shortest distance where the Lennard-Jones interaction of that atomic group with an identical atomic group is zero. The LennardJones parameters were extracted from the specification of the force field.

The grid thus constructed stretched, in the normal direction, between the points where the density of water starts to deviate from its bulk value. The number of grid elements in each direction was chosen such that the linear size of an element was $0.075 \mathrm{~nm}$ on the average. As the area of the bilayer decreases and the thickness increases with an increasing cholesterol content, ${ }^{22}$ the number of elements had to be set separately for each system with a different cholesterol concentration. For instance in the case of pure DPPC, the total area of the bilayer is $41.9 \mathrm{~nm}^{2}$ and the distance between the points where the density of water no longer corresponds to the bulk value is $5.8 \mathrm{~nm}$. With $29.7 \%$ cholesterol, the corresponding values are $26.9 \mathrm{~nm}^{2}$ and $6.4 \mathrm{~nm}$. In the case of pure DPPC, the number of grid elements in the $\mathrm{x}$ and $\mathrm{y}$ directions was taken to be 86 . The number of elements in the normal or $\mathrm{z}$ direction was 78. The corresponding values for the bilayer with $29.7 \%$ cholesterol were 74 and 82 .

We also used finer grids to make sure the results were not influenced by the resolution. In turns out that when the linear size of a grid element is reduced to $0.05 \mathrm{~nm}$, all quantities we have computed change only slightly. The changes are clearly within the error bars originating from a finite simulation time and correlated samples.

In constructing the grids, in the spirit of Ref. 9, we took into account the finite size of small solutes diffusing in bilayers. Due to the finite size, not all grid elements outside the van der Waals radii of the atoms can be accessed by the center of mass (CM) of the diffusing solute. Therefore, in addition to the empty free volume, i. e., all the free volume outside the van der Waals radii of the atoms, we also studied the so-called accessible free volume. Accessible free volume is calculated by adding the van der Waals radius of the diffusing solute to the van der Waals radii of the atoms in the bilayer, and corresponds to the free volume which is accessible to the $\mathrm{CM}$ of the diffusing solute. Several solute sizes with radii $r$ ranging between 0 and $0.2 \mathrm{~nm}$ were studied. For comparison, the effective van der Waals radii of bare sodium, chloride, and potassium ions, as well as those of water and oxygen molecules, are between 0.1 and $0.2 \mathrm{~nm}$. The same applies for example to the general anesthetic Xenon.

The resulting grids were used to compute the average free volume fraction as a function of the distance from the bilayer center. From these it was easy to extract the total free volume in each system. The grids were also the starting point for studying the properties of voids for each system.

\section{B. Identifying and Characterizing Voids}

To characterize the distribution of voids, we need to identify all voids, that is, all clusters of free volume, in our grid. Computing and characterizing the distribution of contiguous clusters of occupied or unoccupied sites is a well-known problem, usually solved by a union / find approach ${ }^{32,43}$ It works as follows. The sites, in this case the empty grid elements, must be traversed. Whenever we arrive at a new site, a find operation is performed, i. e., we check which clusters the nearest neighbor sites, if unoccupied, belong to. The union operation follows: if there are unoccupied nearest neighbor sites that belong to different clusters, those clusters are amalgamated. Otherwise, no union of two clusters is needed.

Modern union/find algorithms are weighted, tree-based, and use path compression. ${ }^{32,43}$ Each cluster is stored as a tree such that the nodes of the tree are the sites of the cluster. Every cluster has a root that corresponds to the root node of the tree, and the other nodes have pointers to either the root or to another node in the same tree. By following a succession of such pointers we can locate the root of any tree. The find operation therefore consists of traversing trees by following pointers to the root nodes. If the nearest neighbor sites of a new site lead to the same root node, the sites belong to the same cluster. The find operation can be implemented such that after the traversal is completed, the pointers along the path all point directly to the root node. This is called path-compression, and it speeds up future traversals. The union of two clusters is achieved by adding a pointer from the root node of one tree to the root of the other. Hence, one of the trees becomes a subtree of the other. This is done in weighted fashion such that the smaller tree becomes a subtree of the larger tree, which is easily accomplished by storing the size of each cluster in the corresponding root node.

Even though the above algorithm may seem a little complicated, it can be implemented, using recursion, in a very elegant and concise manner ${ }^{32}$ Moreover, its performance is in most cases far superior ${ }^{32}$ to simple relabeling algorithms such 
as that introduced in Ref. 44. We chose therefore to adapt the weighted tree-based union-find code with path compression given in Ref. 32 .

The end result of the union / find algorithm for a given configuration is a tree structure containing the information on how the unoccupied sites with known coordinates are connected to each other. From the tree structure we constructed arrays where each array element corresponds to a free volume cluster or void and contains the relevant properties of the void. Such properties are the volume of the void, the location of its CM, and its maximum and minimum coordinates in each direction. Further, we stored the covariance matrix extracted from the coordinates of the grid elements that belong to the void. The covariance matrix can be used to characterize the shape and orientation of the void, as described in the next section.

\section{Principal Component Analysis}

The shape and orientation of the voids were studied using principal component analysis (PCA) ${ }^{33}$ PCA is a versatile statistical method, which is widely used in analysis and compression of multivariate data, signal processing, and neural computing. Here we shall confine ourselves to the case of three-dimensional data.

In applying PCA, we view each void as a three-dimensional ellipsoidal cloud. The principal component vectors are orthogonal and represent the directions with maximum variability. In other words, the principal components define the axes of the ellipsoidal void such that the first principal component is the longest axis, the second is the next longest axis, and the third is the shortest axis.

The principal components may be computed as follows. For a void consisting of $N$ grid elements with coordinates $\left\{\mathbf{r}_{i}\right\}_{i=1}^{N}$, we first compute the mean (or CM) $\mathbf{R}$ and the covariance matrix $\mathbf{C}$. The diagonal of the covariance matrix contains the variances of the Cartesian components of $\mathbf{r}_{i}$. The off-diagonal matrix elements represent correlations between the different components of $\mathbf{r}_{i}$. The covariance matrix is symmetric, and by finding its eigenvalues and eigenvectors we find an orthogonal set of basis vectors. By ordering the eigenvalues, we obtain an orthogonal basis with the first eigenvector having the direction of the largest variance of the data and so on. This is the set of uncorrelated principal components, and simultaneously, the axes of our ellipsoidal void. The square roots of the eigenvalues, in turn, are proportional to the lengths of the axes.

The shape of a void can be deduced from the eigenvalues $\sigma_{i}^{2}$ of the covariance matrix. We chose to focus on the ratios of the square roots of the eigenvalues, denoted by $\sigma_{i} / \sigma_{j}$. In case $\sigma_{1} / \sigma_{2} \approx \sigma_{2} / \sigma_{3} \approx 1$, the void is spherical, otherwise it is elongated. The principal components were used to calculate the orientation of each elongated or non-spherical void. We chose to regard all voids with $\sigma_{1} / \sigma_{3}<1.5$ as spherical. Even though this is not a very strict definition of sphericity, most voids appeared to be non-spherical. Further, this definition was found to be robust, since changing the somewhat arbitrary 1.5 to lower values had virtually no effect on the number of spherical clusters. For the elongated voids, we monitored the angle $\phi$ between the longest axis and the bilayer normal.

As for the practical details of the PCA calculation, we computed the eigenvectors and eigenvalues using singular value decomposition. Further, we only used PCA for voids that were larger than $4 \times 10^{-3} \mathrm{~nm}^{3}$ and smaller than $0.13 \mathrm{~nm}^{3}$. The very small voids were rejected, because it is somewhat artificial to discuss the shape of an object consisting of one or two rectangular grid elements. The large voids were not considered, because their shapes were, in most cases, not ellipsoidal, but rather more complicated with branches and compartments. Applying PCA to these voids would have been meaningless.

\section{Four Region Model}

The bilayer is highly heterogeneous in the normal direction, and both the free volume fraction and the properties of the voids are anticipated to vary with the position along the bilayer normal ${ }^{9}$ It is therefore not sensible to study the void distribution averaged over the whole bilayer, but to discretize the bilayer into regions with more homogeneous compositions. The properties of voids can then be studied in these separate regions. We chose to analyze the data in terms of the four region model introduced by Marrink et al 9.14

The original partition into four regions was slightly modified to better probe the effects of cholesterol on the properties of voids, the main modification being that Region 3 was taken to be the part of the bilayer where the density of cholesterol steroid ring structures is high. We expect the effects of cholesterol on the voids to be most prominent in this region, as the steroid rings rigidify the disordered tails of the DPPC molecules, and enhance the molecular packing. 22.28

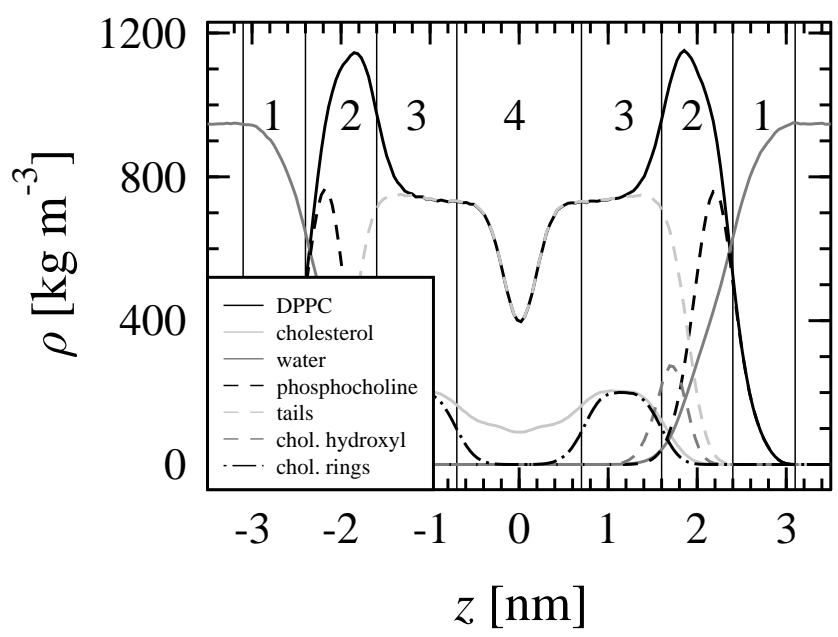

FIG. 1: Mass density profiles of molecules and atomic groups at $\chi=20.3 \%$. The mass density of the cholesterol hydroxyl groups has been scaled by a factor of ten. The division into four regions, indicated here by numbers $1-4$, is based on the mass density profiles, see Sect.IIID

Figure 1 illustrates how we have divided the bilayer into four distinct regions. Region 1 ranges from the point where 
the density of water starts to deviate from the bulk value to the point where the densities of DPPC and water are equal. This region therefore contains mostly water molecules and a low density of DPPC headgroups. Region 2 extends from the point where the densities of water and DPPC coincide to the point where the density of cholesterol ring structures is half of its maximum plateau value ${ }^{22}$ This region contains mainly parts of DPPC molecules: the choline, phosphate, and glycerol densities peak in this region, and there is also a finite density of DPPC tails. Further, the hydroxyl groups of cholesterol reside mainly in this region. Region 3 is defined between the points where the cholesterol steroid ring density assumes half of its maximum value, and contains in addition the middle parts of the DPPC tails. The remaining part of the bilayer is Region 4, i. e., this region is in the center of the bilayer. Here we expect to find a low density of DPPC and cholesterol tails, with the end methyl groups being mainly located in this region.

The boundaries between the regions vary with cholesterol concentration. This is due to the fact that an increasing cholesterol content changes the densities of the various components such that they are shifted away from the bilayer center ${ }^{22}$ Hence, in the case of pure DPPC, the boundaries from the outer boundary of Region 1 to the boundary between Regions 3 and 4 were set to $2.9 \mathrm{~nm}, 2.1 \mathrm{~nm}, 1.4 \mathrm{~nm}$, and $0.6 \mathrm{~nm}$ from the bilayer center. With $29.7 \%$ cholesterol, the corresponding values were $3.2 \mathrm{~nm}, 2.5 \mathrm{~nm}, 1.7 \mathrm{~nm}$, and $0.8 \mathrm{~nm}$.

\section{E. On Percolation Theory}

Percolation theory can be used to predict certain properties of clusters of occupied or unoccupied sites or bonds in a lattice ${ }^{45}$ The applications of percolation theory range from modeling forest fires or the distribution of oil and natural gas in the porous rock in oil reservoirs to studying, say, voids in lipid bilayers. The properties of the clusters are studied as a function of the fraction of occupied or unoccupied sites or bonds, depending on the application. In our case, we are interested in the free volume fraction

$$
p \equiv \frac{\left\langle V_{\text {free }}\right\rangle}{\left\langle V_{\text {tot }}\right\rangle},
$$

where $\left\langle V_{\text {free }}\right\rangle$ is the average free volume in a bilayer and $\left\langle V_{\text {tot }}\right\rangle$ is the total volume of the bilayer. An important free volume fraction is the percolation threshold $p_{\mathrm{c}}$, at which there is a free volume cluster stretching in the $\mathrm{x}, \mathrm{y}$, or $\mathrm{z}$ direction from one side of the system to the opposite side. The size distribution of clusters depends heavily on $p$ and the vicinity of the percolation threshold. At $p \geq p_{c}$, there is a free volume cluster that spans the whole bilayer, and a large fraction of all empty grid elements belong to this percolating cluster. When $p<p_{c}$, there is no such percolating cluster.

The detailed form of the size distribution of clusters cannot, in most cases, be predicted analytically. ${ }^{45} \mathrm{~A}$ general scaling form for the size distribution has been postulated, and the non-universal details such as the form of the scaling function can be extracted from computer simulations. In certain lim- iting cases, however, the exact form of the scaling function becomes irrelevant ${ }^{45}$

Very close to the percolation threshold $p_{\mathrm{c}}$ or for very small clusters, the size distribution $N(V)$ scales with the cluster volume as ${ }^{45}$

$$
N(V) \sim V^{-\tau} .
$$

Here $\tau$ is a universal scaling exponent, which in three dimensions assumes the value $2.18{ }^{45}$ This scaling law is valid for clusters whose linear size is smaller than the correlation length $\xi$, which can be thought of as proportional to a typical cluster diameter. At the percolation threshold $\xi$ approaches infinity, and Eq. (2) is valid for all cluster sizes.

Away from $p_{\mathrm{c}}$ and for large clusters the size distribution scales as follows: ${ }^{45}$

$$
N(V) \sim \begin{cases}V^{-\theta} \exp \left(-c^{\prime \prime} V\right) & p<p_{\mathrm{c}} \\ V^{-\theta^{\prime}} \exp \left(-c^{\prime \prime \prime} V^{1-1 / d}\right) & p>p_{\mathrm{c}}\end{cases}
$$

Here $d$ is the dimensionality of the system, $c^{\prime \prime}$ and $c^{\prime \prime \prime}$ are proportional to $p-p_{\mathrm{c}}$, and $\theta$ and $\theta^{\prime}$ are universal exponents. In three dimensions $\theta=3 / 2$ and $\theta^{\prime}=-1 / 9 .^{45}$ This scaling form applies for clusters whose linear size is larger than $\xi$, in practice for almost all clusters in systems that are not in the near vicinity of $p_{\mathrm{c}}$. Because of the $p-p_{\mathrm{c}}$ dependence of $c^{\prime \prime}$ and $c^{\prime \prime \prime}$, further away from $p_{\mathrm{c}}$ the crossover from power-law to exponential behavior is observed at smaller values of $V$.

We have computed size distributions for systems with different cholesterol concentrations and penetrant sizes. In interpreting the size distribution data, it is useful to know how close to the percolation threshold a given system is. As opposed to infinite systems, in a finite system where the linear dimension in the $\mathrm{z}$ direction cannot be increased, only apparent percolation thresholds can be calculated. $\stackrel{9}{\text { Computing ap- }}$ parent percolation thresholds is not a problem, since this is the threshold a permeant solute will experience. ${ }^{9}$ The apparent percolation threshold can be defined as the free volume fraction at which at least $50 \%$ of all systems percolate ${ }^{\frac{9}{2}}$ Alternatively, $p_{\mathrm{c}}$ can be extracted from maxima in the second moment of the cluster size distribution, computed over all clusters but the percolating one $\mathbb{4 5}^{45}$

Unfortunately, in bilayer systems we cannot automatically expect Eqs. (2) and (3) with three-dimensional critical exponents to describe the behavior of the size distribution at and off the apparent percolation threshold. Predicting the form of the size distribution is in fact much more difficult for bilayer systems than for isotropic bulk systems in two or three dimensions. First, the bilayer is heterogeneous in the normal direction, and consequently, the free volume fraction varies with the position along the bilayer normal, i. e., $p=p(z)$, see Fig. 4 The bilayer center or Region 4 , with the highest free volume fraction in the whole bilayer, ${ }^{9,22}$ could be well above the percolation threshold, while the other regions are well below $p_{\mathrm{c}}$. This problem can be in part remedied by considering the four regions with approximately constant free volume fractions. Secondly, the $\mathrm{z}$ direction is very different from the $\mathrm{x}$ and y directions, and there might well be different thresholds for percolation in different directions. Finally, there are finite 


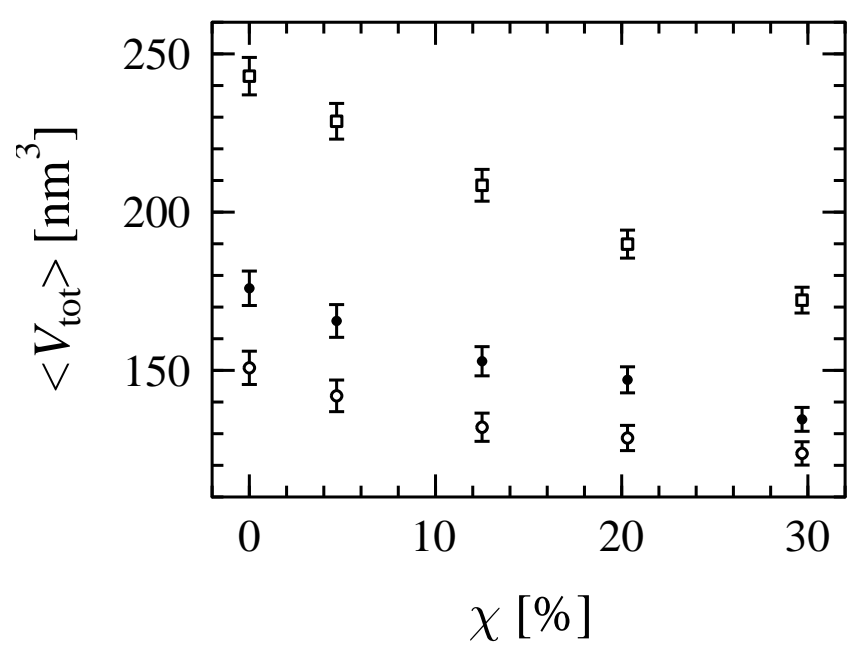

FIG. 2: Bilayer volume as function of cholesterol concentration. As there is no unique definition for the thickness of a bilayer, the thicknesses have been measured in three ways: between the points where the mass density of water starts to deviate from the bulk value ( $\square$ ), between the points where the mass density of phospholipids and water are equal (o), and between the maxima in the total electron densities $(\bullet)$.

size effects. The dimension in the normal direction will seldom exceed $10 \mathrm{~nm}$ in bilayer systems. This makes the system quasi-two-dimensional rather than three-dimensional.

\section{RESULTS}

\section{A. Total Volume and Free Volume}

It is believed that the total free volume in a lipid bilayer decreases when the cholesterol concentration increases ${ }^{4.7}$ On the other hand, it is often assumed that the total volume of the bilayer is kept approximately constant with a growing cholesterol concentration. This is attributed to the decreasing area and increasing thickness of the bilayer. As a cholesterol occupies less volume than a DPPC, the volume occupied by phospholipids and cholesterols must diminish with an increasing cholesterol concentration. Water is pushed away from the bilayer center ${ }^{22}$ and hence we expect that the volume occupied by water molecules should at least not grow. The constant total volume and a reduced volume occupied by cholesterol, DPPC molecules, and water would together suggest that the free volume should, contrary to the general belief, increase. To determine whether the total free volume decreases or increases, we have computed the total volume and total free volume as functions of cholesterol concentration.

The total volume is computed by multiplying the average area of the bilayer by the thickness of the bilayer. Because of the rough interface between the bilayer and the water phase, there is no unique definition for the thickness of the bilayer. As measures of thickness we have used the distance between the points where the mass density of water starts to deviate from the bulk density, between the points where the mass den- sities of phospholipids and water coincide, and between the maxima in the total electron densities ${ }^{22}$ The total volume as a function of the cholesterol concentration is shown in Fig. 2 It is clear that regardless of how we define the thickness of the bilayer, the total volume decreases with a growing cholesterol concentration.

Unfortunately, there is little experimental evidence to either confirm or contradict this result. To our knowledge, there are no systematic studies on the effect of cholesterol on the phospholipid bilayer area or volume. Monolayer studies do exist, ${ }^{46}$ but the applicability of these results to bilayers is questionable 47.48

As the total volume and the occupied volume both decrease with an increasing cholesterol concentration, it is not immediately clear how the free volume should be expected to behave. This issue can be resolved by extracting the total free volumes from the three-dimensional grids introduced in Sect. IIIA The procedure can be thought of as integrating over the total free volumes in thin slices in the xy plane. The range of integration must be the thickness of the bilayer, defined as in the case of total volume. The results are shown in Fig. 3 It is clear that the total free volume decreases with an increasing cholesterol concentration, notwithstanding how the thickness is measured. We can therefore conclude that both the occupied and free volume decrease, together resulting in a reduction of the total volume of the bilayer.

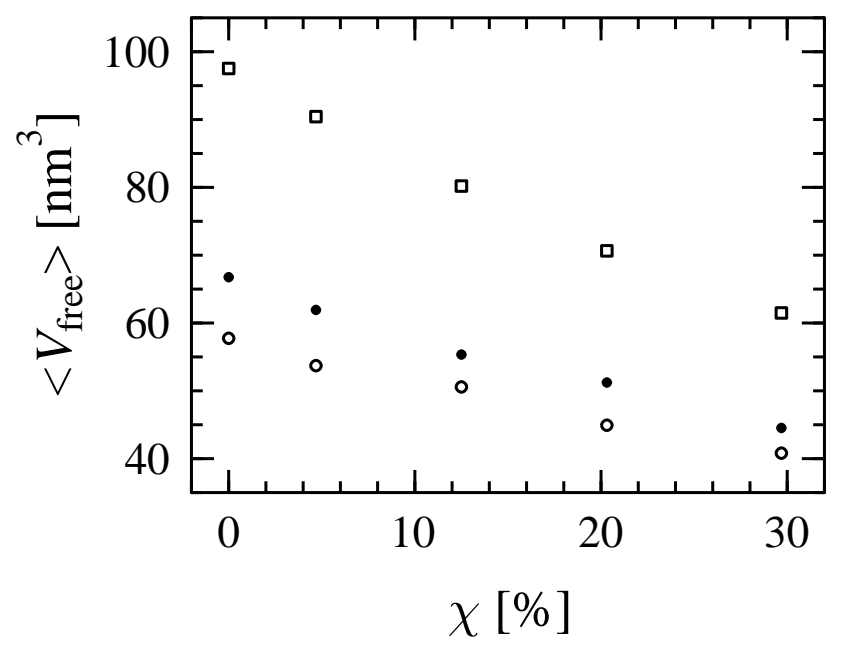

FIG. 3: Empty free volume as function of cholesterol concentration. The bilayer thicknesses have been defined as the distance between the points where the density of water starts to deviate from the bulk value $(\square)$, the distance between the points where the density of phospholipids and water are equal (o), and the distance between the maxima in the total electron densities $(\bullet)$. The errors are of the order of a few per cent.

Experimental findings are in favor of the reduction of free volume. Galla et al $\stackrel{7}{?}$ have reached this conclusion by comparing their phospholipid diffusion coefficients to the predictions of free area theory. Almeida et $\mathrm{al}^{4}{ }^{4}$ present empirical laws for the behavior of specific volumes and thicknesses of DMPC/cholesterol bilayers as functions of temperature. Based on these laws they have computed the average areas 
per molecule at different cholesterol concentrations. Combining these with close-packed areas for DMPC and cholesterol molecules, they have estimated the average free areas per phospholipid. The free areas thus obtained explain nicely the diffusion coefficients from fluorescence recovery after photobleaching (FRAP) experiments. ${ }^{4}$

\section{B. Free Volume Fraction Profile}

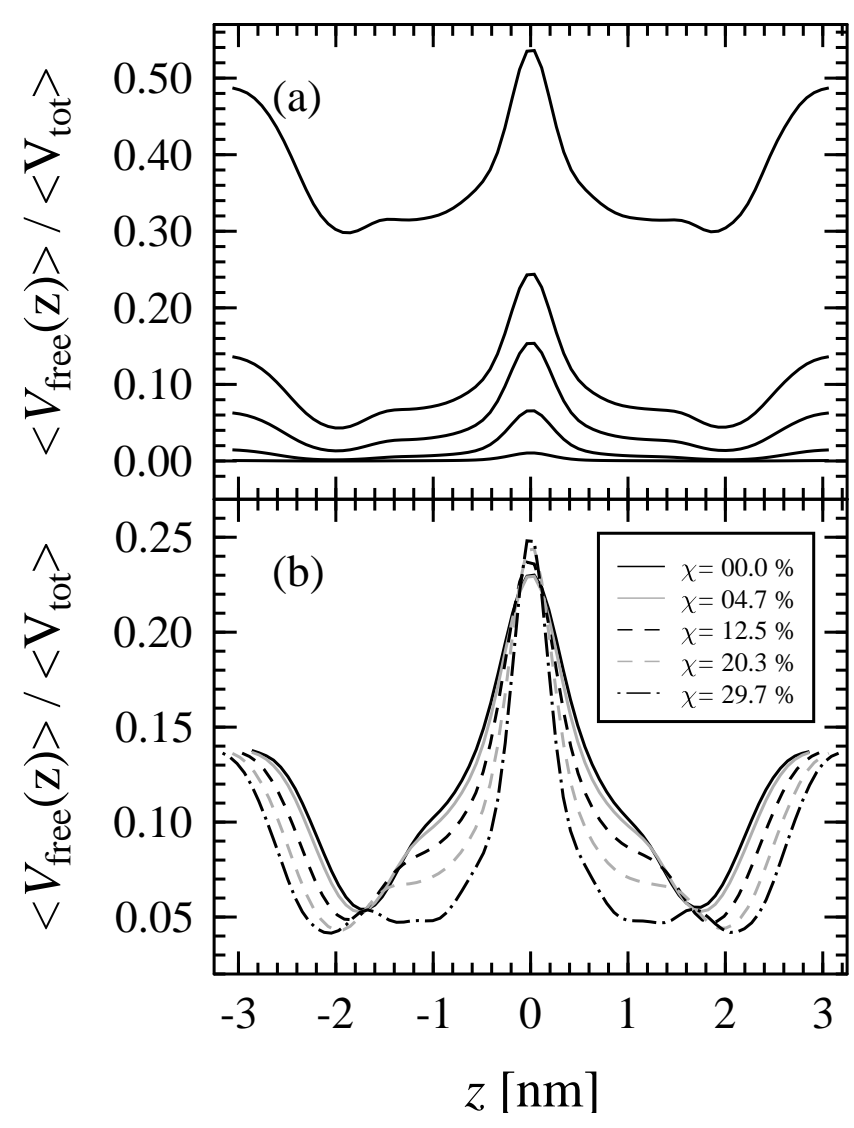

FIG. 4: Free volume fractions as functions of position along bilayer normal. (a) Empty free volume fraction and accessible free volume fractions for bilayer with $20.3 \%$ cholesterol. The penetrant radii from top to bottom are $0.00 \mathrm{~nm}, 0.05 \mathrm{~nm}, 0.07 \mathrm{~nm}, 0.10 \mathrm{~nm}$, and $0.15 \mathrm{~nm}$. (b) Accessible free volume fraction for different cholesterol concentrations for a penetrant with a radius $r=0.05 \mathrm{~nm}$. In both panels the value $z=0$ corresponds to the bilayer center, and the errors are of the order of a percent.

Having concluded that cholesterol reduces the total free volume, let us now look at the behavior of free volume in different parts of the bilayer. Free volume fraction profiles, i. e., free volume fractions as functions of the position $z$ along the bilayer normal, are displayed in Fig. 4 Part (a) of the figure illustrates the effect of penetrant size on the free volume fraction with $20.3 \%$ cholesterol. The uppermost curve is the empty free volume fraction, while the bottom one corresponds to the accessible free volume fraction for penetrants with a radius $r=0.15 \mathrm{~nm}$. Part (b) shows the effect of choles- terol concentration on the accessible free volume fraction with $r=0.05 \mathrm{~nm}$.

All free volume fraction profiles in Fig. 4 regardless of the penetrant size or cholesterol concentration, have maxima in the bilayer center and minima in Region 2. The penetrant size has a strong effect on free volume fraction: at least a third of the total volume at each $z$ seems to be empty free volume, while for penetrants with $r=0.15 \mathrm{~nm}$, apart from the bilayer center, there is virtually no accessible free volume at all. These findings are in good qualitative agreement with the results of Marrink et al., 9 considering that those simulations were performed at a temperature $T=350 \mathrm{~K}$ and that temperature has a significant effect on free volume $\frac{5}{\underline{5}}$ Cholesterol influences the shape of the profile, reducing the free volume fractions in Regions 1 and 3. The decrease in Region 3 must be due to the presence of the rigid steroid ring structure. In Region 1, the origin of the effect may be the reorientation of the DPPC headgroups. 22

\section{Percolation}



FIG. 5: Second moments of void size distributions computed over all but percolating cluster as functions of penetrant radii. The open circles are for $\chi=0 \%$ and the filled ones for $\chi=29.7 \%$. The errors are of the order of a few percent.

Examining the nature and location of the percolation transition is essential for a proper interpretation of the size distributions. We have located the percolation threshold by computing the second moment of the size distribution of voids, see Sect. IIIE The second moments for $\chi=0 \%$ and $29.7 \%$ as functions of penetrant radii are shown in Fig. 5 Since a maximum indicates percolation, we can identify two percolation transitions for each cholesterol concentration. For small $r$ percolation takes place in $\mathrm{x}, \mathrm{y}$, and $\mathrm{z}$ directions. In between the two maxima the system percolates in the plane of the bilayer, but no longer in the normal direction. This xy percolation always takes place in Region 4. Both of these transitions are important from a biological point of view: the xyz percolation is relevant for permeation across the bilayer, whereas $x y$ percolation in the bilayer center could be significant, e.g., for 
diffusion of quinone within the bilayer. Finally, at large values of $r$ there is no percolation.

The location of the transitions depends weakly on cholesterol concentration. The xyz percolation takes place at $r=$ $0.048(2) \mathrm{nm}$ when $\chi=0 \%$ and at $r=0.044(2) \mathrm{nm}$ when $\chi=29.7 \%$. The corresponding values for the $\mathrm{xy}$ percolation are $r=0.080(2) \mathrm{nm}$ and $r=0.073(2) \mathrm{nm}$. A larger fraction of cholesterol therefore seems to imply that slightly smaller penetrants perceive the system as percolating.

As for percolation thresholds, it is meaningless to define anything such for the xyz percolation, since the free volume fraction varies strongly along the bilayer normal. The xy percolation in the more homogeneous Region 4 is quite another matter. In this case we can, using plots similar to Fig. 4 map the penetrant sizes for which percolation takes place to free volume fractions. The threshold for xy percolation is $p=0.06$ at all cholesterol concentrations. Cholesterol does not alter the percolation threshold itself, but it changes slightly the free volume fraction for a given penetrant size. This causes the weak $\chi$ dependence of the location of the xy percolation seen in Fig. 5 The value of 0.06 is in good agreement with the corresponding threshold calculated by Marrink et al. for pure DPPC and thus equals their value for soft polymer as well 9

\section{Size Distributions}

The size distributions are unnormalized to better illustrate the behavior of the absolute number of voids in a bilayer of a given composition. Therefore $N(V)$ is the average number of voids of volume $V$. As the four regions have very distinct material properties, ${ }^{9.14 .15}$ we have considered the size distributions separately in each region. Further, the vicinity of the percolation transitions is significant for the form of the distribution. We have identified two cases that represent the most biologically relevant regimes: $r=0.05 \mathrm{~nm}$ at which xy percolation takes place in Region 4 and $r=0.09 \mathrm{~nm}$ with no percolation. The regime where xyz percolation occurs is quite similar to the case of $r=0.05 \mathrm{~nm}$. Besides, even though the results presented here are qualitative rather than quantitative in nature, describing the qualitative effect of cholesterol instead of claiming to establish accurate numerical values, penetrant radii smaller than $0.05 \mathrm{~nm}$ are unphysical.

The effect of cholesterol on the void size distributions is largely restricted to those parts where the cholesterol molecules are located. In Region 1 , regardless of $r$, cholesterol has no impact on the size distributions. This is not surprising, since no part of cholesterol is present in the water phase. The effects are still very minor in Region 2, although the hydroxyl groups of cholesterol are mainly located in this region. The situation in Regions 3 and 4, where the cholesterol steroid ring structures and tails are situated, is illustrated in Fig. 6 These are somewhat coupled, since a particularly large cluster could extend to both regions, but is assigned to Region 4, as its CM is located there.

The behavior of these two regions with an increasing cholesterol concentration depends on $r$. The case of $r=$ $0.05 \mathrm{~nm}$, representing all $r$ below the xyz percolation thresh-

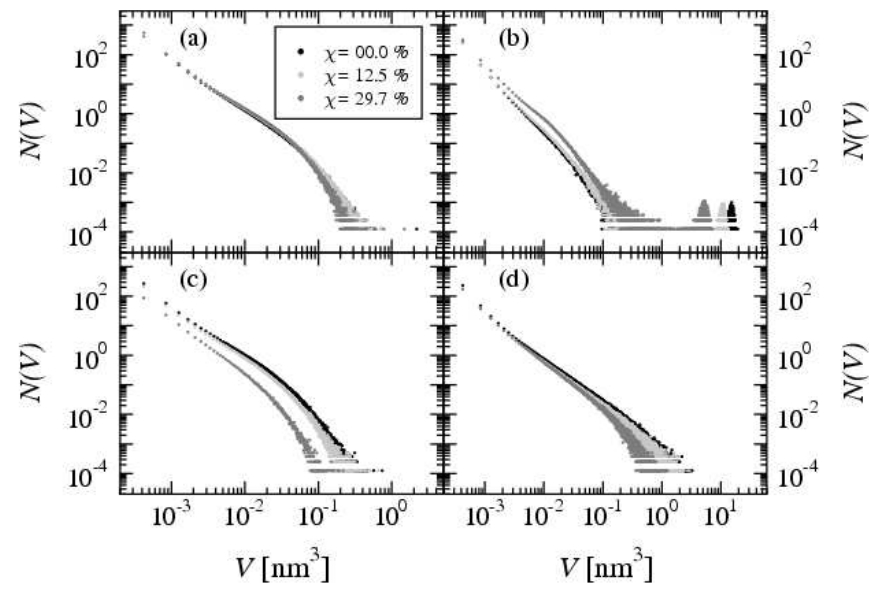

FIG. 6: Effect of cholesterol concentration on void size distribution. (a) Region 3 and $r=0.05 \mathrm{~nm}$. (b) Region 4 and $r=0.05 \mathrm{~nm}$. (c) Region 3 and $r=0.09 \mathrm{~nm}$. (d) Region 4 and $r=0.09 \mathrm{~nm}$. Percolation in the plane of the bilayer is seen in panel (b) as a separate cluster of points in the right-hand corner. The finite statistics lead to relative errors that grow with $V$. For $V<0.01 \mathrm{~nm}^{3}$ the errors in $N(V)$ are smaller than a percent, and in the range $0.01 \mathrm{~nm}^{3}<V<$ $0.1 \mathrm{~nm}^{3}$ smaller than ten percent. If $V$ is of the order of $1 \mathrm{~nm}^{3}$, the relative errors may be $100-300 \%$. As the data are shown on a $\log \log$ scale, this is hardly a problem.

old and above the xy threshold, is portrayed in panels (a) and (b) of Fig. 6 The most notable effect of replacing $29.7 \%$ of the DPPC molecules by cholesterols is the decreasing connectivity of free volume. This is manifested in the size of the percolating cluster, which decreases by a factor of three to $V \approx$ $5 \mathrm{~nm}^{3}$, approximately ten times the average close-packed volume of a cholesterol molecule, $V_{\text {chol }} \approx 0.459(2) \mathrm{nm}^{3} .22$ When connectivity is reduced, the number of non-percolating voids of all sizes in Region 4 increases: although the total free volume decreases, the number of voids increases approximately by a factor of three. The effects in Region 3 are more minor. Here the number of voids from $V$ with radii of $0.1 \mathrm{~nm}$ to $V$ of the order of a tenth of $V_{\text {chol }}$ increases very slightly. The number of large voids with $V$ close to $V_{\text {chol }}$ decreases somewhat.

The situation is quite different when no percolating cluster is present, as illustrated by results computed with $r=0.09 \mathrm{~nm}$ in panels (c) and (d) of Fig. 6 The effects of cholesterol are very pronounced in Region 3. Comparing the case of $29.7 \%$ cholesterol to pure DPPC we note that there are fewer voids of all sizes. The number of voids is reduced at least by a factor of three, but especially for larger voids the reduction can be as large as a factor of twenty. The largest voids with $0.2 \mathrm{~nm}^{3} \lesssim V \lesssim 0.7 \mathrm{~nm}^{3}$, i.e., voids with sizes close to $V_{\text {chol }}$, are removed completely. The effects are similar, although more minor, in Region 4. Voids with radii up to $0.1 \mathrm{~nm}$ are unaffected, while the number of intermediate and large voids decreases. Here cholesterol removes the voids with $1 \mathrm{~nm}^{3} \lesssim V \lesssim 3 \mathrm{~nm}^{3}$, larger than its own close-packed size.

Let us finally analyze Fig. 6 from the point of view of percolation theory. Below the xyz percolation threshold, i. e., for 
both $r=0.05 \mathrm{~nm}$ and $r=0.09 \mathrm{~nm}$, there is no percolating cluster in Region 3. If we assume that bulk three-dimensional percolation theory holds, in Region 3 we expect to observe power-law behavior with an exponent $\theta=1.5$ at small and intermediate $V$ and cross-over to exponential behavior at large $V$. This indeed seems to be the case, see panels (a) and (c). We also note that the cross-over to exponential behavior occurs at smaller $V$ for $r=0.09 \mathrm{~nm}$. This is to be anticipated, since the larger the penetrant radius, the further away from the xyz percolation threshold we are. In the case of Region 4 with $r=0.09 \mathrm{~nm}$, see panel (d), we are very close to the xy percolation threshold. At very small $V$, power-law behavior with an exponent close to 2.1 is observed. The linear sizes of these small voids must hence be smaller than the correlation length $\xi$. At intermediate and large $V$, the power-law behavior can still be observed, with an exponent close to 1.5. Pure DPPC, which is closer to the percolation threshold than bilayers with finite cholesterol concentrations, shows no cross-over to exponential behavior, while with finite $\chi$ we observe exponential behavior for the largest $V$. The behavior in Region 4 above the xy percolation threshold, see panel (b), is difficult to interpret, as we are in between two percolation thresholds. At small and intermediate $V$ we again observe power-law behavior with an exponent close to 2.2. At larger values of $V$, the situation is less clear.

Having already mentioned the impact of penetrant size and region on the void size distributions, let us discuss them at some more length. Void size distributions at $\chi=20.3 \%$ in Region 3 for penetrants with radii ranging from $0.05 \mathrm{~nm}$ to $0.15 \mathrm{~nm}$ are displayed in Fig. 7 a). We can immediately draw the obvious conclusion that large penetrants see fewer and smaller voids than small ones. As Region 3 with $r \geq 0.05 \mathrm{~nm}$ is below any percolation threshold, all cases look very similar: power-law behavior with an exponent 1.5 at small and intermediate $V$ and a cross-over to exponential behavior at larger $V$. The cross-over moves to smaller $V$ with an increasing $r$, as we are getting further away from the xyz percolation threshold.

The effect of region on the case of $\chi=20.3 \%$ and $r=0.09 \mathrm{~nm}$ is shown in Fig. (7) b). The smallest number of voids, as well as the smallest voids, can be found in the tightly packed headgroup region or Region 2. As expected, Region 4 features much larger voids than any other region. All curves shown in this figure agree well with the predictions of the bulk three-dimensional percolation theory below the percolation threshold.

\section{E. Shape}

Using PCA we can extract $\sigma_{1}, \sigma_{2}$, and $\sigma_{3}$, which are proportional to the lengths of the principal axes of an ellipsoidal void. From these we can compute $P\left(\sigma_{1} / \sigma_{2}, \sigma_{2} / \sigma_{3}\right)$, a distribution describing the probability that a void has given values of $\sigma_{1} / \sigma_{2}$ and $\sigma_{2} / \sigma_{3}$. The distribution has been normalized such that integration over it gives unity. As visualizing and comparing different $P\left(\sigma_{1} / \sigma_{2}, \sigma_{2} / \sigma_{3}\right)$ can be difficult, we have also computed $P\left(\sigma_{i} / \sigma_{j}\right)$ describing the probability of

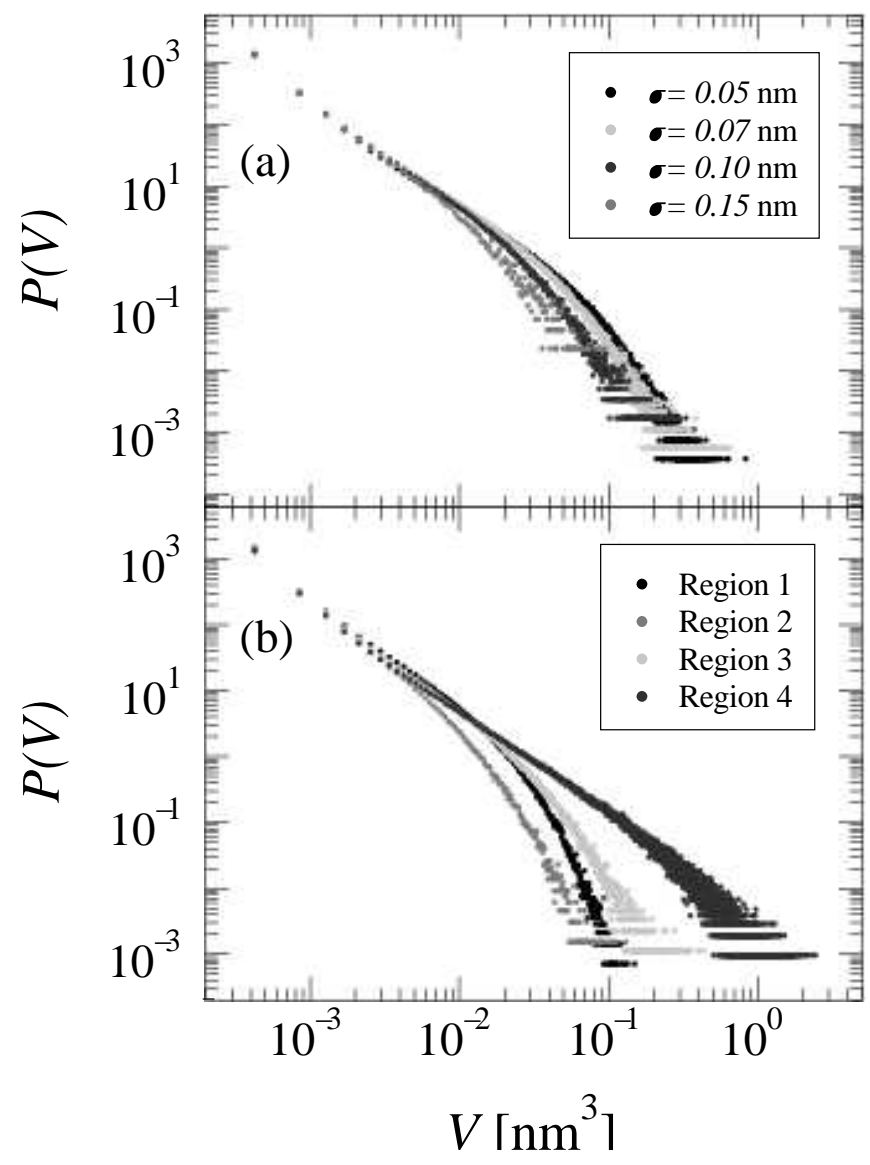

FIG. 7: Effect of penetrant size and region on void size distribution. (a) Size distributions for different penetrant sizes for the case $\chi=$ $20.3 \%$ and Region 3. (b) Size distributions in different regions for $\chi=20.3 \%$ and $r=0.09 \mathrm{~nm}$. The relative errors are similar to those in Fig. 6

finding a void with a given value of $\sigma_{i} / \sigma_{j}$ irrespective of $\sigma_{k}$, $k \neq i, j$. These distributions have been scaled such that integration over them gives unity.

Interpretation of the probability distributions will give us information on the shape of the voids for which $4 \times 10^{-3} \mathrm{~nm}^{3}$ $<V<0.13 \mathrm{~nm}^{3}$, see Sect. IIIC If $\sigma_{1} / \sigma_{2} \approx \sigma_{2} / \sigma_{3} \approx 1$, the void is spherical. In case $\sigma_{1} \gg \sigma_{2} \approx \sigma_{3}$, we are dealing with a roughly cigar-shaped void. Finally, if $\sigma_{1} \approx \sigma_{2} \gg \sigma_{3}$, our void is essentially disk-like.

The effect of cholesterol on $P\left(\sigma_{1} / \sigma_{2}, \sigma_{2} / \sigma_{3}\right)$ in Region 3 with $r=0.05 \mathrm{~nm}$ is portrayed in Fig. 8 Cholesterol does not influence the shape of the voids in any other region, and other penetrant sizes give very similar results (data not shown). Panels (a) and (b) show the probability distribution for pure DPPC and panels (c) and (d) for a bilayer with $29.7 \%$ cholesterol. In both cases elongated voids dominate the distributions: spherical or nearly spherical voids are rare. The presence of cholesterol makes the voids more elongated, i. e., larger values of $\sigma_{1} / \sigma_{2}$ and $\sigma_{2} / \sigma_{3}$ occur with a higher probability.

The elongating effect of cholesterol can be further studied by focusing on $P\left(\sigma_{1} / \sigma_{2}\right)$ and $P\left(\sigma_{2} / \sigma_{3}\right)$ shown in panels (a) and (b) of Fig. 9 These figures show a systematic average 
(a)

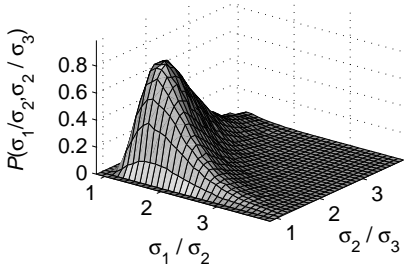

(c)

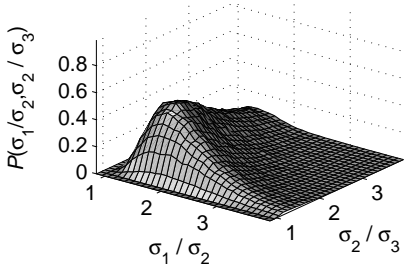

(b)

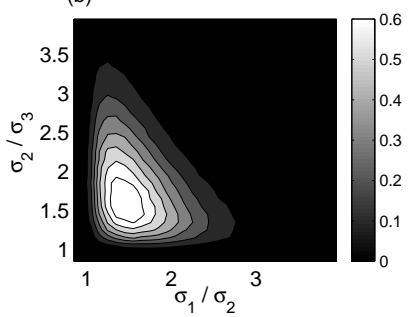

(d)

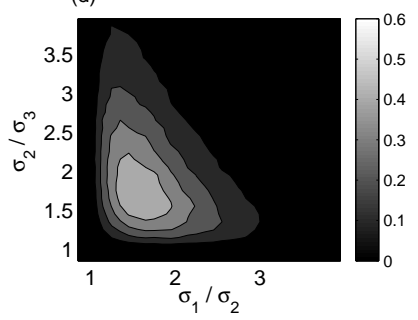

FIG. 8: $P\left(\sigma_{1} / \sigma_{2}, \sigma_{2} / \sigma_{3}\right)$ at $\chi=0 \%$ and $\chi=29.7 \%$ in Region 3. The penetrant size is $0.05 \mathrm{~nm}$. (a) Surface plot at $\chi=0.0 \%$. (b) Contour plot at $\chi=0.0 \%$. (c) Surface plot at $\chi=29.7 \%$. (d) Contour plot at $\chi=29.7 \%$. The relative errors are smaller than ten percent.

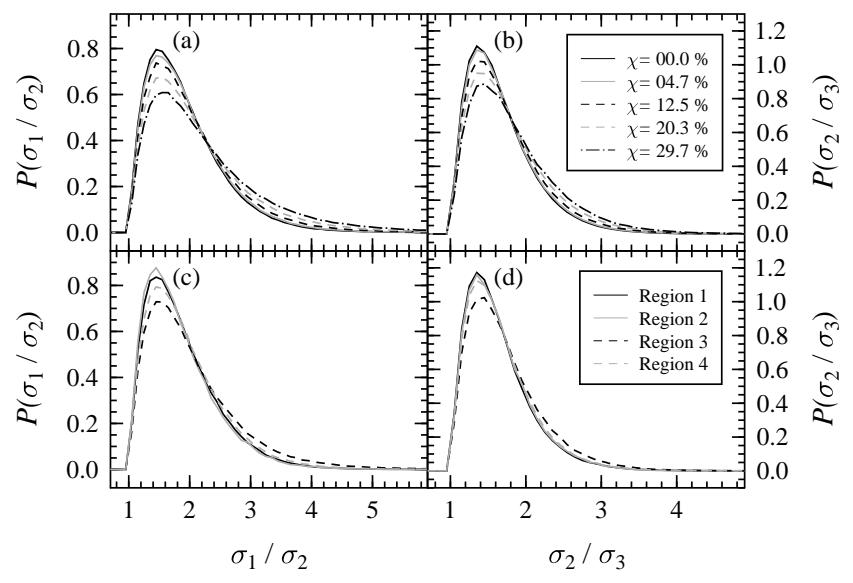

FIG. 9: $P\left(\sigma_{i} / \sigma_{j}\right)$ at different cholesterol concentrations and in different regions. (a) $P\left(\sigma_{1} / \sigma_{2}\right)$ in Region 3 for $r=0.05 \mathrm{~nm}$. (b) $P\left(\sigma_{2} / \sigma_{3}\right)$ in Region 3 for $r=0.05 \mathrm{~nm}$. (c) $P\left(\sigma_{1} / \sigma_{2}\right)$ for $\chi=20.3 \%$ and $r=0.09 \mathrm{~nm}$. (d) $P\left(\sigma_{2} / \sigma_{3}\right)$ for $\chi=20.3 \%$ and $r=0.09 \mathrm{~nm}$. The relative errors are smaller than two per cent when $\sigma_{i} / \sigma_{j}<3$, and between two and five percent for $3<\sigma_{i} / \sigma_{j}<6$.

elongation with an increasing cholesterol content: the larger the concentration, the more elongated voids we see.

Figure 9 also depicts the behavior of $P\left(\sigma_{1} / \sigma_{2}\right)$ and $P\left(\sigma_{2} / \sigma_{3}\right)$ in different regions, see panels (c) and (d). In this case the bilayer contains $20.3 \%$ cholesterol and $r=0.09 \mathrm{~nm}$, which represents well all penetrant sizes. Regions 1 and 2 are very similar to each other. Most voids larger than the lower cutoff $V=4 \times 10^{-3} \mathrm{~nm}^{3}$ have been taken into account in PCA, since the vast majority of voids is smaller than the upper cutoff $V=0.13 \mathrm{~nm}^{3}$, see Fig. 7] Region 4 appears to differ from Regions 1 and 2 only slightly. This is true for small voids with $V<0.13 \mathrm{~nm}^{3}$. We should, however, keep in

mind that Region 4 also contains larger voids, see Fig. 7 The shapes of these holes are not ellipsoidal but more complex. The voids in Region 3 are more elongated than elsewhere. When the cholesterol concentration increases, this effect becomes slightly more pronounced. If $\chi=0 \%$, all regions look approximately similar.

\section{F. Orientation}

The orientations of the principal axes of the ellipsoidal voids can be extracted from PCA. We have measured the cosine of the angle $\phi$ between the longest axis of an elongated ellipsoid and the bilayer normal. When the longest axis is oriented along the bilayer normal $\phi=0$. In principle, $\phi \in[0, \pi)$, but due to the symmetry properties of ellipsoids the angles $\phi$ and $\pi-\phi$ are equivalent. We will therefore confine ourselves to $\phi \in[0, \pi / 2]$. To describe the orientation of the voids, we have computed the quantity $P(\cos \phi)$, the probability that a given void is oriented such that the cosine of the angle between its longest axis and the bilayer normal is $\cos \phi$. The probability distribution has been normalized to give unity when integrated over, $\int_{0}^{1} \mathrm{~d}(\cos \phi) P(\cos \phi)=1$. Should we wish to think in terms of $\phi$ rather than $\cos \phi$, averages are computed by integrating over $\phi$ and the weight of a given angle is $P(\cos \phi) \sin \phi$.

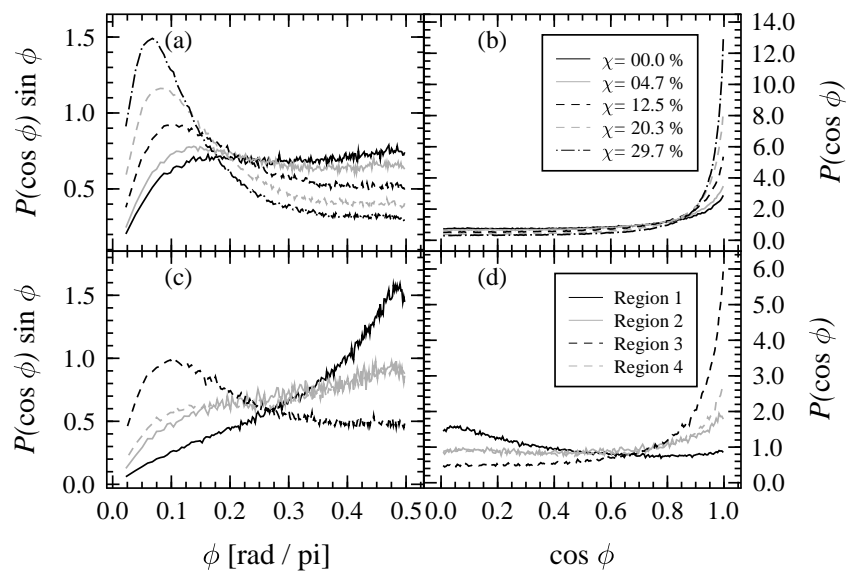

FIG. 10: Orientational distributions at different cholesterol concentrations and in different regions. (a) $P(\cos \phi) \sin \phi$ in Region 3 for $r=0.05 \mathrm{~nm}$. (b) $P(\cos \phi)$ in Region 3 for $r=0.05 \mathrm{~nm}$. (c) $P(\cos \phi) \sin \phi$ for $\chi=20.3 \%$ and $r=0.09 \mathrm{~nm}$. (d) $P(\cos \phi) \phi$ for $\chi=20.3 \%$ and $r=0.09 \mathrm{~nm}$. The relative errors are of the order of five percent for both $P(\cos \phi)$ and $P(\cos \phi) \sin \phi$.

The impact of cholesterol concentration on $P(\cos \phi)$ and $P(\cos \phi) \sin \phi$ depends on which region of the bilayer we study. There are hardly any changes in either Region 1 or 2. The effect of cholesterol is strongest in Region 3, and largely independent of $r$. The case of $r=0.05 \mathrm{~nm}$ is portrayed in panels (a) and (b) of Fig. 10 In pure DPPC, angles $\phi>0.1 \pi$ are favored, while orientation along the bilayer normal hardly occurs at all. At $\chi=29.7 \%$, the angle with the largest weight is $\phi=0.06 \pi$. Orientation along the bilayer normal or close to 
it is favored, and perpendicular orientation is rare. At cholesterol concentrations between 0 and $29.7 \%$, the orientational distributions change systematically from one extreme to the other. The presence of cholesterol can therefore be said to promote orientation along the bilayer normal in Region 3.

In Region 4 the effects of cholesterol are less pronounced and depend on $r$ (data not shown). Between the xy and xyz percolation thresholds, e.g., in the case of $r=0.05 \mathrm{~nm}$, orientation along the bilayer normal is favored. An increasing cholesterol concentration seems to accentuate this effect. Below the xy percolation threshold, for $r=0.09 \mathrm{~nm}$ and larger, perpendicular orientation is favored and few voids are oriented along the bilayer normal, see panel (c) of Fig. 10 Cholesterol has a minor effect here, suppressing the weight of intermediate angles $\phi \approx 0.25 \pi$ and making perpendicular orientational slightly more probable. As discussed in the end of Sect.IVF it should be kept in mind that these distributions have been computed for voids smaller than $V=0.13 \mathrm{~nm}^{3}$. For instance in the case of $r=0.05 \mathrm{~nm}$, Region 4 contains a large percolating cluster of a complex shape, oriented more or less in the plane of the bilayer.

The situation in different regions at $\chi=20.3 \%$ and for $r=0.09 \mathrm{~nm}$ is portrayed in panels (c) and (d) of Fig. 10. The effect of changing $\chi$ and $r$ has been described above. In Regions 1 and 2, the orientational distribution of voids is very broad with a peak close to $\phi=\pi / 2$. Angles near $\phi \approx 0$ hardly occur at all. In Region 3, orientation along the bilayer normal or close to it is favored. Region 4, as described above, contains mostly voids that are oriented perpendicular to the bilayer normal.

\section{G. Size, Shape, and Orientation}

So far we have considered the shape and orientation of all voids irrespective of their size. In this section we shall investigate whether voids of different sizes differ in average shape or orientation. For this purpose we have computed $P\left(V, \sigma_{1} / \sigma_{2}\right)$, the probability of finding a void of volume $V$ with a given value of $\sigma_{1} / \sigma_{2}$, and $P(V, \cos \phi)$, the probability of finding a void of volume $V$ with a given $\cos \phi$. Both probability distributions have been normalized such that integration over them results in unity.

Unfortunately, $P\left(V, \sigma_{1} / \sigma_{2}\right)$ and $P(V, \cos \phi)$ are quite noisy and extremely difficult to visualize. Therefore we have calculated the average values of $\sigma_{1} / \sigma_{2}$ and $\cos \phi$ for each $V$, defined as follows:

$$
\begin{aligned}
\left\langle\sigma_{1} / \sigma_{2}\right\rangle_{V} & \equiv \frac{\int_{1}^{\infty} \mathrm{d}\left(\sigma_{1} / \sigma_{2}\right) P\left(V, \sigma_{1} / \sigma_{2}\right) \sigma_{1} / \sigma_{2}}{\int_{1}^{\infty} \mathrm{d}\left(\sigma_{1} / \sigma_{2}\right) P\left(V, \sigma_{1} / \sigma_{2}\right)}, \\
\langle\cos \phi\rangle_{V} & \equiv \frac{\int_{0}^{1} \mathrm{~d}(\cos \phi) P(V, \cos \phi) \cos \phi}{\int_{0}^{1} \mathrm{~d}(\cos \phi) P(V, \cos \phi)} .
\end{aligned}
$$

These might still be somewhat noisy, as the number of voids of a given size $V$ may be quite small.

In analyzing $\left\langle\sigma_{1} / \sigma_{2}\right\rangle_{V}$ and $\langle\cos \phi\rangle_{V}$, we shall focus on the effects of cholesterol in Region 3 and for $r=0.05 \mathrm{~nm}$. Based on our studies of $P\left(\sigma_{1} / \sigma_{2}\right), P(\cos \phi)$, and related quantities, we expect the impact of cholesterol to be most significant in this region. Further, we do not anticipate our results to change significantly with $r$.

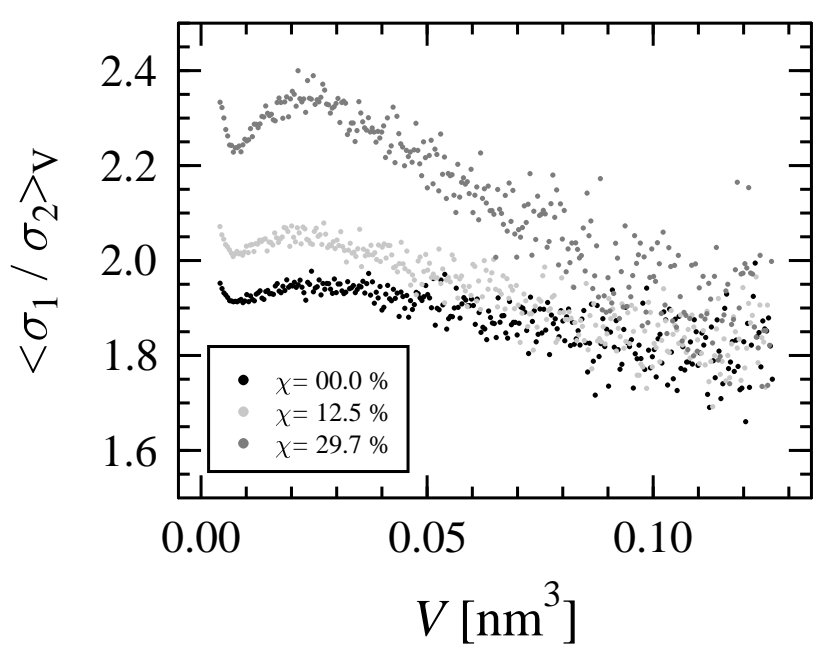

FIG. 11: Averages $\left\langle\sigma_{1} / \sigma_{2}\right\rangle_{V}$ as functions of $V$ in Region 3 and for $r=0.05 \mathrm{~nm}$. The relative errors grow with $V$ : for $V<0.05 \mathrm{~nm}^{3}$ the errors are smaller than three percent, for $0.05 \mathrm{~nm}^{3}<V<$ $0.10 \mathrm{~nm}^{3}$ they grow linearly in the range $4-10 \%$, and for larger $V$ they can be up to $20 \%$.

The behavior of $\left\langle\sigma_{1} / \sigma_{2}\right\rangle_{V}$ with $V$ is shown in Fig. 11 In the case of pure DPPC, our data indicate that voids of all sizes should have approximately similar shapes. With $29.7 \%$ cholesterol small voids with $V<V_{\text {chol }} / 10$ seem to be more elongated than larger ones. The shapes of large voids are little affected by an increasing cholesterol concentration. Based on these data, we can conclude that the presence and amount of cholesterol appear to mainly affect the shape of small voids.

Figure 12 depicts the effect of cholesterol on $\langle\cos \phi\rangle_{V}$ vs. $V$. At all $\chi$, the voids become on the average slightly more perpendicularly oriented with an increasing $V$. An increasing cholesterol concentration makes voids of all sizes adopt average orientations closer to the direction of the bilayer normal. We can therefore conclude that an increasing cholesterol concentration affects the orientation of at least the voids with $4 \times 10^{-3} \mathrm{~nm}^{3}<V<0.13 \mathrm{~nm}^{3}$.

\section{H. Local Effects}

We have seen that a finite cholesterol concentration changes the properties of voids in a bilayer. It is natural to ask whether or not the changes are more pronounced in the close vicinity of cholesterol molecules. In principle, investigating the locality of the changes cholesterol brings about is simple: we just have to single out the voids whose CMs are located near the $\mathrm{CM}$ of a cholesterol molecule, and compare their properties to voids whose CMs are nowhere near cholesterols. There are, however, a few catches that should be avoided.

First, studying arbitrarily large voids is meaningless. The $\mathrm{CM}$ of a very large void may or may not be close to the $\mathrm{CM}$ of 


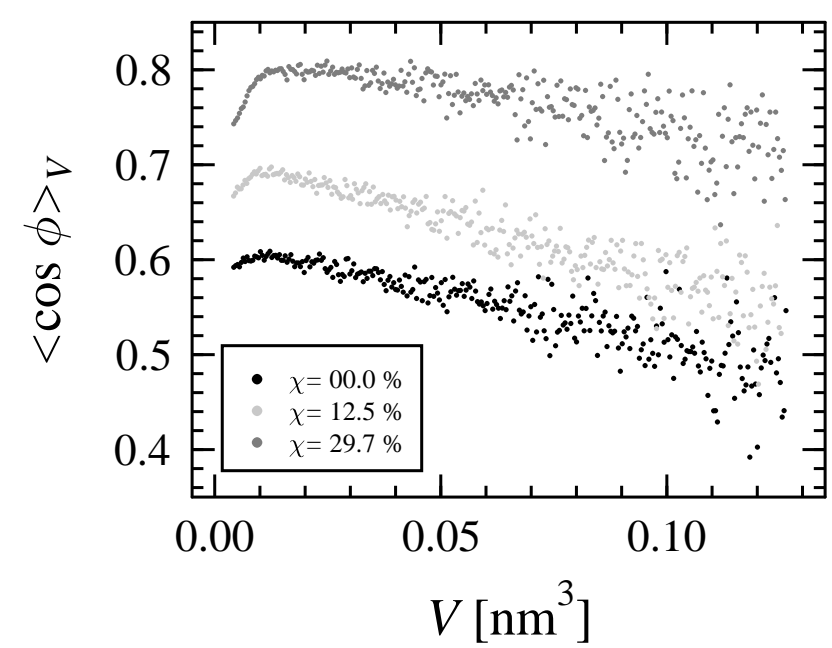

FIG. 12: Averages $\langle\cos \phi\rangle_{V}$ as functions of $V$ in Region 3 and for $r=0.05 \mathrm{~nm}$. The relative errors grow with $V$ : for $V<0.01 \mathrm{~nm}^{3}$ the errors are smaller than a percent, for $0.01 \mathrm{~nm}^{3}<V<0.05 \mathrm{~nm}^{3}$ smaller than five percent, for $0.01 \mathrm{~nm}^{3}<V<0.05 \mathrm{~nm}^{3}$ smaller than ten percent, and for larger $V$ up to $25 \%$.

a cholesterol, even though some parts of the void might be in the vicinity of one or several cholesterols. In any event, such large clusters cannot very well be said to be localized. Hence, we have confined ourselves to voids that are smaller than a cholesterol molecule, i. e., voids with $V<V_{\text {chol }}$.

An additional problem is choosing a suitable criterion for the proximity of a void to a cholesterol molecule. The criterion should not be too strict, leading to automatic exclusion of all large and perpendicularly oriented voids from the class of voids close to cholesterol. This can be ascertained by monitoring the principal radii of voids and choosing the criterion based on these. The choice can be facilitated by restricting the study to Region 3, where the voids will, by definition, be in the same region as the steroid ring structures. This allows us to consider distances that are projected to the plane of the bilayer.

We have chosen to focus on concentration $\chi=20.3 \%$, and the penetrant radius has been set to $r=0.10 \mathrm{~nm}$. After investigating the principal axes of voids in this case, we have decided to regard voids with $\mathrm{CMs}$ within $0.8 \mathrm{~nm}$ of a $\mathrm{CM}$ of a cholesterol molecule, making sure that both are in the same monolayer, as proximate to cholesterol. This decision is based on two observations: the radius corresponding to the thickest part of a cholesterol molecule in our study is approximately $0.3 \mathrm{~nm}, 22$ and the vast majority of voids in our current setup have principal radii smaller than $0.5 \mathrm{~nm}$.

The results are shown in Fig. 13 Panel (a) features the size distributions computed for voids in the vicinity of cholesterols and for those far from cholesterols. To facilitate comparison, these distributions have been normalized by the average number of voids close to cholesterols in Region 3 and by the average number of voids far from cholesterol in Region 3. The resulting normalized distribution $P(V)$ is such that it gives unity when integrated over. Voids with radii smaller than $0.1 \mathrm{~nm}$


FIG. 13: Effect of proximity of cholesterol molecules at $\chi=20.3 \%$ with $r=0.10 \mathrm{~nm}$. The data for voids close to cholesterols are shown in black and those for voids far from cholesterols in gray. (a) Normalized void size distributions. (c) $P\left(\sigma_{1} / \sigma_{2}\right)$. (b) Orientational distributions.

are unaffected by the proximity of cholesterol. Larger voids, however, are affected: the vicinity of cholesterol reduces the probability of finding a void with $V \approx V_{\text {chol }} / 10$ by a little less than a factor of ten. Further, near cholesterols there are no voids with $0.1 \mathrm{~nm}^{3}<V<V_{\text {chol }}$.

Is the size distribution for voids far from cholesterol coincident with the size distribution for Region 3 of a pure DPPC bilayer? Considering voids with $V<V_{\text {chol }}$ and normalizing by the total number of voids in each case, we obtain distributions that can be compared directly. It turns out that the two are nearly identical. Hence, further away from cholesterols the size distribution of voids is unaffected by the presence of cholesterol and practically identical to the corresponding distribution in pure DPPC.

The effect of the proximity of cholesterol molecules on void 
shape is portrayed in panel (b) of Fig. 13. It is evident that the shape of voids does not depend on the vicinity of cholesterols. Orientation of voids near cholesterols and far from them is portrayed in panel (c) of the same figure. The voids that are located close to cholesterols tend to be more oriented along the bilayer normal, while the ones far from cholesterols show a broader orientational distribution.

\section{CONCLUDING DISCUSSION}

We have studied the impact of cholesterol on the voids in simulated bilayers consisting of DPPC. Using data from atomistic molecular dynamics simulations, we have investigated the behavior of the total free volume with an increasing cholesterol concentration, extracted the void size distributions in different parts of the bilayer, and considered the effect of cholesterol on the shape and orientation of the voids. We have also probed whether or not the effects of cholesterol on the properties of voids are localized to the near vicinity of cholesterol molecules. In interpreting the data, we have, in the spirit of Ref. 9, made use of three-dimensional isotropic percolation theory.

Our findings support the reduction of the total free volume with an increasing cholesterol content. The effects of cholesterol on individual voids appear to be the most prominent in those parts of the bilayer where cholesterol's steroid ring structures and the DPPC tails are located. Here cholesterol reduces the connectivity of free volume, thus altering the void size distributions. These changes are perceptible already at low cholesterol concentrations and become pronounced with approximately $20 \%$ cholesterol in the bilayer. For instance a concentration of $29.7 \%$ leads to complete removal of the voids that are approximately of the same size as a cholesterol molecule. An increasing cholesterol concentration also makes the voids in the region containing the steroid rings more elongated, and transforms the isotropic orientational distribution of voids present in pure DPPC to an anisotropic one favoring orientation of voids along the bilayer normal. All these effects appear to be localized to the proximity of cholesterol molecules, rather than being uniformly distributed to the whole bilayer.

Reasons for the changes in the region with the cholesterol ring structures and the hydrocarbon tails of DPPCs can be found in previous computational studies on DPPC/cholesterol systems 22.28 .49 .50 .51 Cholesterol increases the order parameters of the hydrocarbon tails by simultaneously reducing their tilt and the number of gauche-defects present in them. The straightening of the tails should affect both the void size distributions and, in particular, the shape and orientation of the voids. This explanation is supported by the fact that the effects of cholesterol are confined to the proximity of cholesterol molecules: the effect of cholesterol on the ordering of the phospholipid tails is much stronger in the vicinity of cholesterol molecules 52 In addition to the ordering, there is another, more obvious factor, which should certainly influence the void size distributions. The bulky cholesterol molecule simply fills empty spaces of its own size in the bilayer, as suggested by Almeida et al..$^{4}$

Our results for pure DPPC are in good qualitative agreement with the findings of Marrink et al.,$^{\frac{9}{9}}$ although their study has been performed at $T=350 \mathrm{~K}$ and is based on $80 \mathrm{ps}$ of data. The form of our void size distributions in different regions and for different permeants is in surprisingly good agreement with theirs. Their measure for the shape of the voids is different from ours, not enabling them to fully distinguish between elongated and fractal voids. In addition, they study voids of all sizes, while we have restricted our studies on the shape and orientation of voids to the range $4 \times 10^{-3} \mathrm{~nm}^{3}$ $<V<0.13 \mathrm{~nm}^{3}$. Their qualitative conclusion is that larger voids may be more elongated and/or more fractal than small ones. We, on the other hand, find that the shape of the voids depends only weakly on the size, and that the voids become slightly less elongated with an increasing volume. As for orientation, despite the different measures, their conclusions are in accord with ours. They observe that the orientational distribution of voids is most anisotropic is in Region 3, while Region 1 features an almost completely isotropic distribution.

We also agree with Marrink et al ${ }^{9}$ in that the complexity of the properties of the voids in a phospholipid bilayer imply that simple free area theories cannot be used for quantitative predictions. However, free volume arguments may be used for qualitatively explaining diffusion and permeation in bilayers. An increasing cholesterol concentration in DPPC bilayers leads to monotonously decreasing lateral diffusion coefficients for both DPPCs and cholesterols. ${ }^{22}$ Based on our current findings on the properties of voids, this is to be expected. Intuitively, smaller and fewer voids, which are oriented along the bilayer normal, should slow down lateral diffusion of lipids and sterols. The details of what role the voids play for the various diffusion and permeation mechanisms in bilayers are challenging questions that large-scale simulation studies may elucidate in the near future.

In addition to the static properties of voids considered in this study, also the dynamic properties should be important to dynamic processes in bilayers. It would be interesting to study the dynamics of void formation and the movement of individual voids. Such studies should give us further insight into mechanisms of lateral diffusion and solute permeation in membranes.

\section{Acknowledgments}

We are grateful for Teemu Murtola and Mikko Alava for fruitful discussions. This work has, in part, been supported by the Academy of Finland through its Center of Excellence Program (E. F., I. V.), the National Graduate School in Materials Physics (E. F.), the Academy of Finland Grant Nos. 54113, 00119 (M. K.), 80246 (I. V.), and 80851 (M. H.), and the Jenny and Antti Wihuri Foundation (M.H.). M. P. would like to acknowledge the support through the Marie Curie fellowship No. HPMF-CT-2002-01794. We would also like to thank the Finnish IT Center for Science and the HorseShoe (DCSC) supercluster computing facility at the University of Southern Denmark for computer resources. 
${ }^{1}$ B. Alberts, D. Bray, J. Lewis, M. Raff, K. Roberts, and J. D. Watson, Molecular Biology of the Cell (Garland Publishing, New York, 1994), 3rd ed.

2 M. Bloom, E. Evans, and O. G. Mouritsen, Q. Rev. Biophys. 24, 293 (1991).

${ }^{3}$ M. N. Jones and D. Chapman, Micelles, Monolayers, and Biomembranes (Wiley-Liss, New York, 1995).

${ }^{4}$ P. F. F. Almeida, W. L. C. Vaz, and T. E. Thompson, Biochemistry 31, 6739 (1992).

5 D. Bassolino-Klimas, H. E. Alper, and T. R. Stouch, J. Am. Chem. Soc. 117, 4118 (1995).

6 A. Bondi, J. Phys. Chem. 58, 929 (1954).

7 H.-J. Galla, W. Hartmann, U. Theilen, and E. Sackmann, J. Membrane Biol. 48, 215 (1979).

8 C. Loison, M. Mareschal, and F. Schmid, J. Chem. Phys. 121, 1890 (2004).

9 S. J. Marrink, R. M. Sok, and H. J. C. Berendsen, J. Chem. Phys. 104, 9090 (1996).

${ }^{10}$ K. Rajagopal and V. Sitaramam, J. Theor. Biol. 195, 245 (1998).

11 J. C. Mathai, Z. E. Sauna, O. John, and V. Sitaramam, J. Biol. Chem. 268, 15442 (1993).

12 D. Bemporad, J. W. Essex, and C. Luttmann, J. Phys. Chem. B 108, 4875 (2004).

13 P. Jedlovszky and M. Mezei, J. Phys. Chem. B 107, 5322 (2003).

${ }^{14}$ S. J. Marrink and H. J. C. Berendsen, J. Phys. Chem. 98, 4155 (1994).

15 S. J. Marrink and H. J. C. Berendsen, J. Phys. Chem. 100, 16729 (1996).

16 T. R. Stouch and D. Bassolino, in Biological Membranes: a Molecular Perspective from Computation and Experiment, edited by K. M. Merz, Jr. and B. Roux (Birkhäuser, Boston, 1996), pp. 255-280.

${ }^{17}$ R. S. Cantor, Biophys. J. 76, 2625 (1999).

${ }_{18}$ R. G. Eckenhoff, Mol. Interv. 1, 258 (2001).

19 J. Repáková, P. Čapková, J. M. Holopainen, and I. Vattulainen, J, Phys. Chem. B (2004), in press.

${ }^{20}$ W. H. de Jeu, B. I. Ostrovskii, and A. N. Shalaginov, Rev. Mod. Phys. 75, 181 (2003).

${ }^{21}$ M. H. Cohen and D. Turnbull, J. Chem. Phys. 31, 1164 (1959).

${ }^{22}$ E. Falck, M. Patra, M. Karttunen, M. T. Hyvönen, and I. Vattulainen, Biophys. J. X, x (2004).

${ }^{23}$ S. Mitragotri, M. E. Johnson, D. Blankschtein, and R. Langer, Biophys. J. 77, 1268 (1999).

24 T.-X. Xiang, J. Phys. Chem. B 103, 385 (1999).

25 D. Bassolino-Klimas, H. E. Alper, and T. R. Stouch, Biochemistry 32, 12624 (1993).

${ }^{26}$ W. Shinoda, M. Mikami, T. Baba, and M. Hato, J. Phys. Chem. B 108, 9346 (2004).
27 M. R. Vist and J. H. Davis, Biochemistry 29, 451 (1990).

28 C. Hofsäß, E. Lindahl, and O. Edholm, Biophys. J. 84, 2192 (2003).

29 J. M. Polson, I. Vattulainen, H. Zhu, and M. J. Zuckermann, Eur. Phys. J. E 5, 485 (2001).

${ }^{30}$ I. Vattulainen and O. G. Mouritsen, in Diffusion in Condensed Matter: Methods, Materials, and Models, edited by D. Heitjans and J. Kärger (Springer-Verlag, Berlin, 2005), 2nd ed., in press.

31 A. Pralle, P. Keller, E.-L. Florin, K.Simons, and J. K. H. Hörber, J. Cell Biol. 148, 997 (2000).

${ }^{32}$ M. E. J. Newman and R. M. Ziff, Phys. Rev. E 64, 016706 (2001).

33 S. Sharma, Applied Multivariate Techniques (Wiley, New York, 1996).

${ }^{34}$ O. Berger, O. Edholm, and F. Jahnig, Biophys. J. 72, 2002 (1997).

${ }^{35}$ M. Höltje, T. Förster, B. Brandt, T. Engels, W. von Rybinski, and H.-D. Höltje, Biophys. Biochim. Acta 1511, 156 (2001).

${ }^{36}$ D. P. Tieleman and H. J. C. Berendsen, J. Chem. Phys. 105, 4871 (1996).

37 E. Lindahl, B. Hess, and D. van der Spoel, J. Mol. Model. 7, 306 (2001).

${ }^{38}$ H. J. C. Berendsen, J. P. M. Postma, W. F. van Gunsteren, A. DiNola, and J. R. Haak, J. Chem. Phys. 81, 3684 (1984).

39 U. Essman, L. Perera, M. L. Berkowitz, H. L. T. Darden, and L. G. Pedersen, J. Chem. Phys. 103, 8577 (1995).

${ }^{40}$ M. Patra, M. Karttunen, M. T. Hyvönen, E. Falck, P. Lindqvist, and I. Vattulainen, Biophys. J. 84, 3636 (2003).

${ }^{41}$ M. Patra, M. Karttunen, M. T. Hyvönen, E. Falck, and I. Vattulainen, J. Phys. Chem. B 108, 4485 (2004).

${ }^{42}$ E. Falck, to be published.

43 T. H. Cormen, C. E. Leiserson, R. L. Rivest, and C. Stein, Introduction to Algorithms (MIT Press and McGraw-Hill, USA, 2001), 2nd ed.

44 J. Hoshen and R. Kopelman, Phys. rev. B 14, 3438 (1976).

45 S. Stauffer and A. Aharony, Introduction to Percolation Theory (Taylor and Francis, London, 1992), 2nd ed.

46 H. M. McConnell and A. Radhakrishnan, Biochim. Biophys. Acta 1610, 159 (2003).

${ }^{47}$ Y. N. Kaznessis, S. Kim, and R. G. Larson, Biophys. J. 82, 1731 (2002).

48 J. F. Nagle and S. Tristram-Nagle, Biochim. Biophys. Acta 1469, 159 (2000)

49 S. W. Chiu, E. Jacobsson, R. J. Mashl, and H. L. Scott, Biophys. J. 83, 1842 (2002).

50 T. Róg and M. Pasenkiewicz-Gierula, Biophys. J. 81, 2190 (2001).

51 A. M. Smondyrev and M. L. Berkowitz, Biophys. J. 77, 2075 (1999).

52 P. Jedlovszky and M. Mezei, J. Phys. Chem. B 107, 5311 (2003). 